\title{
Equilíbrio postural e risco para queda em idosos da cidade de Garça, SP
}

\section{Postural balanceandrisk for fallsineddely inthedity of Garça, stateof SãoPaulo, Brazil}

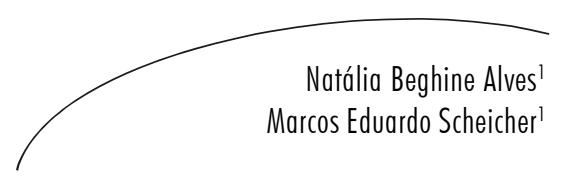

Objetivos: Avaliar o equilíbrio postural e o risco de quedas em idosos institucionalizados e não-institucionalizados. Métodos: Foram incluídos no estudo 45 idosos institucionalizados e 43 idosos não-institucionalizados. O equilíbrio e o risco de quedas foram avaliados através da Escala de Equilíbrio de Berg. A comparação do equilíbrio entre os grupos foi feita por meio do teste de MannWhitney. Foi avaliado o risco para queda entre as populações. Adotou-se um valor de $\mathrm{p} \leq$ 0,05 como significante. Resultados: Houve diferença entre os escores da Escala de Berg nas populações estudadas ( $\mathrm{p}<0,0001$ ). Os resultados mostraram que a população institucionalizada apresenta nove vezes mais risco de cair do que idosos da comunidade $(\mathrm{p}=0,0001)$. Conclusões: Os resultados indicam que o equilíbrio postural é menor na população institucionalizada, aumentando seu risco de cair.

\section{Abstract}

Objectives: To evaluate the postural balance and risk of falls in institutionalized and non-institutionalized elderly. Methods: The study included 45 institutionalized and 43 non-institutionalized elderly. The risk of falls and balance were assessed through the Berg Balance Scale. The comparison of the balance between the groups was performed using the Mann-Whitney test. The risk of falls between populations was evaluated. We adopted a value of $\mathrm{p} \leq 0.05$ as significant. Results: There was a difference between the Berg Scale scores in the populations studied $(p<0.0001)$. The results showed that the institutionalized population has 9 times more likely to fall than elderly of the community $(p=0.0001)$. Conclusions: The results indicate that postural balance is lower in the institutionalized population, increasing their risk of falling.

\footnotetext{
Curso de Fisioterapia, Faculdade de Filosofia e Ciências. Universidade Estadual Paulista - Unesp. Marília, SP, Brasil.
}

Correspondência / Correspondence

Marcos Eduardo Scheicher

UNESP - Departamento de Educação Especial

Av. Hygino Muzzi Filho, 737

17525-900 Marília, SP, Brasil

E-mail: mscheicher@marilia.unesp.br
Palavras-chave: Idoso. Envelhecimento da população. Acidentes por quedas. Equilíbrio postural.
Key words: Elderly. Demographic aging. Accidental falls. Postural balance. 


\section{INTRODUÇÃO}

O envelhecimento populacional é uma realidade no nosso país, assim como em todo mundo. Com o aumento do número de idosos, ocorre uma elevação das doenças associadas ao envelhecimento, destacando-se as crônico-degenerativas.

Uma das consequências mais graves do envelhecimento são as quedas, que são reconhecidas como um importante problema de saúde pública entre os idosos, devido a frequência, morbidade e elevado custo social e econômico decorrente das lesões provocadas.

A queda é um evento frequente e limitante na vida do idoso e é definida como um evento inesperado e não intencional que tem como resultado a mudança de posição do indivíduo para um nível mais baixo em relação à posição inicial que se dá em decorrência da perda total do equilíbrio postural e da ineficiência dos mecanismos necessários à manutenção do controle postural. Como consequência dessa ineficiência, o indivíduo se torna incapaz de corrigir os deslocamentos do corpo durante seu movimento no espaço, resultando em um desequilíbrio que pode culminar em queda. ${ }^{1-5}$

Pessoas de todas as idades apresentam risco de sofrer queda. Porém, para os idosos, elas possuem um significado muito relevante, pois podem leválo à incapacidade, injúria e morte. Seu custo social é imenso e se torna maior quando o idoso tem diminuição da autonomia e da independência ou passa a necessitar de institucionalização. ${ }^{1}$

Os acidentes intra e extradomiciliares são a quinta causa de morte entre os idosos e as quedas são responsáveis por $70 \%$ dessas mortes acidentais. ${ }^{47} \mathrm{~A}$ internação em instituições de longa permanência para idosos (ILPIs) pode ser considerada um fator de risco para quedas, já que a maioria dos idosos institucionalizados apresenta enfermidades que necessitam de atenção e cuidados especiais. Além disso, esses indivíduos tendem a viver em isolamento social e não praticam atividades físicas, debilitando ainda mais sua saúde, o que aumenta as chances de quedas. ${ }^{3}$ Um estudo realizado no sul do Brasil encontrou alta prevalência de quedas em idosos institucionalizados. ${ }^{8}$

As quedas e suas consequências para as pessoas idosas no Brasil têm assumido dimensão de epidemia. ${ }^{9}$ Os custos para a pessoa idosa que cai e faz uma fratura são incalculáveis. E o pior, atinge toda a família, na medida em que a pessoa idosa que fratura um osso acaba hospitalizada e frequentemente é submetida a tratamento cirúrgico. Os custos para o sistema de saúde também são altos. Diante disso, os objetivos do trabalho foram avaliar e comparar o equilíbrio e o risco de quedas em idosos institucionalizados e não-institucionalizados.

\section{MÉTODO}

\section{Sujeitos da amostra}

Foi realizado estudo transversal. Entre os meses de julho de 2009 a março de 2010, foram selecionados 45 idosos moradores de duas instituições, o Lar dos Velhos Frederico Ozanan e o Lar São Vicente de Paulo, da cidade de Garça, SP, e 43 idosos não-institucionalizados, frequentadores de grupos de atividade física, idade acima de 65 anos ou mais, de ambos os sexos, que aceitaram espontaneamente participar da pesquisa. Todos assinaram termo de consentimento livre e esclarecido. A pesquisa foi aprovada pelo Comitê de Ética em Pesquisa da Faculdade de Filosofia e Ciências - UNESPMarília, sob o protocolo n ${ }^{\circ} 1038 / 2008$.

Os seguintes critérios de inclusão foram adotados: ter 65 anos de idade ou mais, residir na comunidade ou em uma instituição para idosos, marcha independente. Os critérios de exclusão foram: prejuízo cognitivo, ter doenças que interferissem no equilíbrio corporal (doenças neuromusculoesqueléticas), problemas visuais não corrigidos e hipotensão postural.

\section{Protocolo}

A avaliação do equilíbrio e do risco de quedas dos idosos foi feita por meio da Escala de 
Equilíbrio de Berg - versão brasileira, validada e adaptada para a língua portuguesa. ${ }^{10}$ Antes da aplicação do teste, as atividades que compõem a avaliação foram demonstradas pelo avaliador.

O teste é composto por 14 itens categorizados numa escala ordinal de cinco pontos ( 0 - incapaz de realizar e 5 - realiza com independência). $\mathrm{O}$ teste avalia tanto a forma como é realizado cada item, assim como o tempo em que este se realiza. Os escores totais variam entre 0 a 56 pontos, sendo que a máxima pontuação está relacionada a um melhor desempenho. Os elementos do teste são representativos de AVDs que, juntas, podem responder sobre o desempenho dos idosos nas atividades motoras, bem como o equilíbrio ao realizá-las, e também podem prever a probabilidade de ocorrência de quedas. Sujeitos com escore igual ou inferior a 45 pontos possuem risco de quedas aumentado..$^{5,10-13}$

\section{Análise estatística}

Os dados antropométricos foram apresentados como média \pm desvio padrão. As análises estatísticas foram realizadas pelo Software GraphPad
Instat, sendo que as comparações entre os grupos de idosos institucionalizados e nãoinstitucionalizados foram feitas por meio do teste de Mann-Whitney. O risco foi calculado pelo Risco Relativo, adotando-se um valor de $\mathrm{p} \leq 0,05$ como significante.

\section{RESULTADOS}

Participaram do estudo 45 idosos institucionalizados e 43 não-institucionalizados, com média de idade de 73,3 $\pm 5,2$ e 71,2 $\pm 4,7$, respectivamente. Do total dos idosos institucionalizados, 27 eram do gênero masculino e 18 do gênero feminino. Dos idosos da comunidade, 14 eram homens e 29 eram mulheres.

A figura 1 mostra os valores individuais e a média dos escores da Escala de Berg para idosos institucionalizados e não-institucionalizados $(35,2$ $\pm 5,6$ e $42,3 \pm 4,4)$.

Houve diferença estatística entre os grupos $(\mathrm{p}<0,0001)$. Percebe-se que os idosos moradores de ILPIs tiveram um desempenho preocupante.

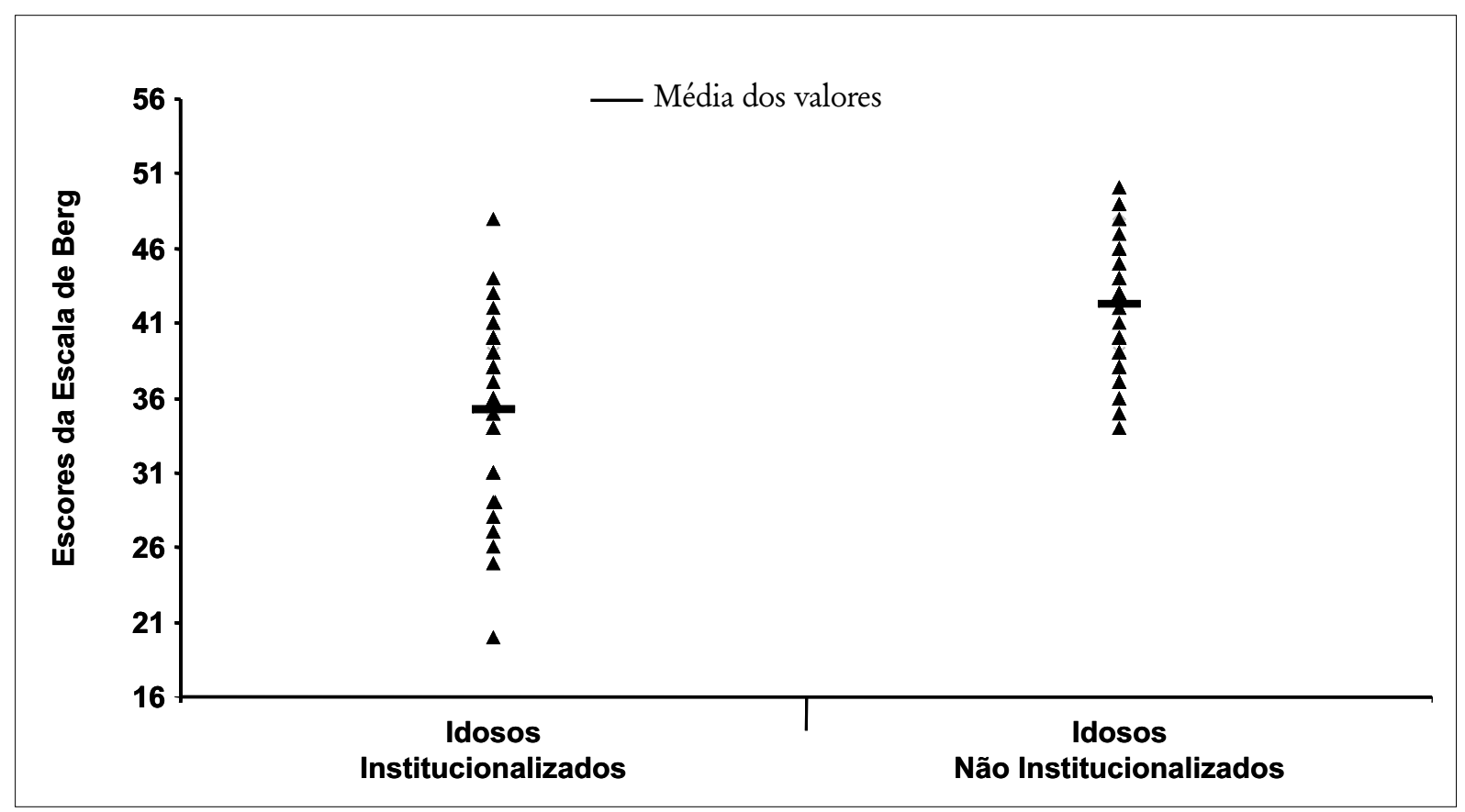

Figura 1 - valores individuais dos escores da Escala de Equilíbrio de Berg para idosos institucionalizados e não institucionalizados. 
A figura 2 indica o número de indivíduos institucionalizados e não-institucionalizados que apresentaram risco de quedas aumentado por meio dos escores obtidos na Escala de Berg. Observa-se que, dos 45 sujeitos institucionalizados, 44 (97,7\%) obtiveram escores menor que 45 pontos, indicando que o risco de quedas nesses idosos está aumentado. ${ }^{5,10-13} \mathrm{Na}$ população nãoinstitucionalizada, 31 idosos apresentaram pontuação igual ou menor que 45 pontos $(R R=$ $9,0)$. Houve diferença estatística entre os grupos $(p<0,0001)$.

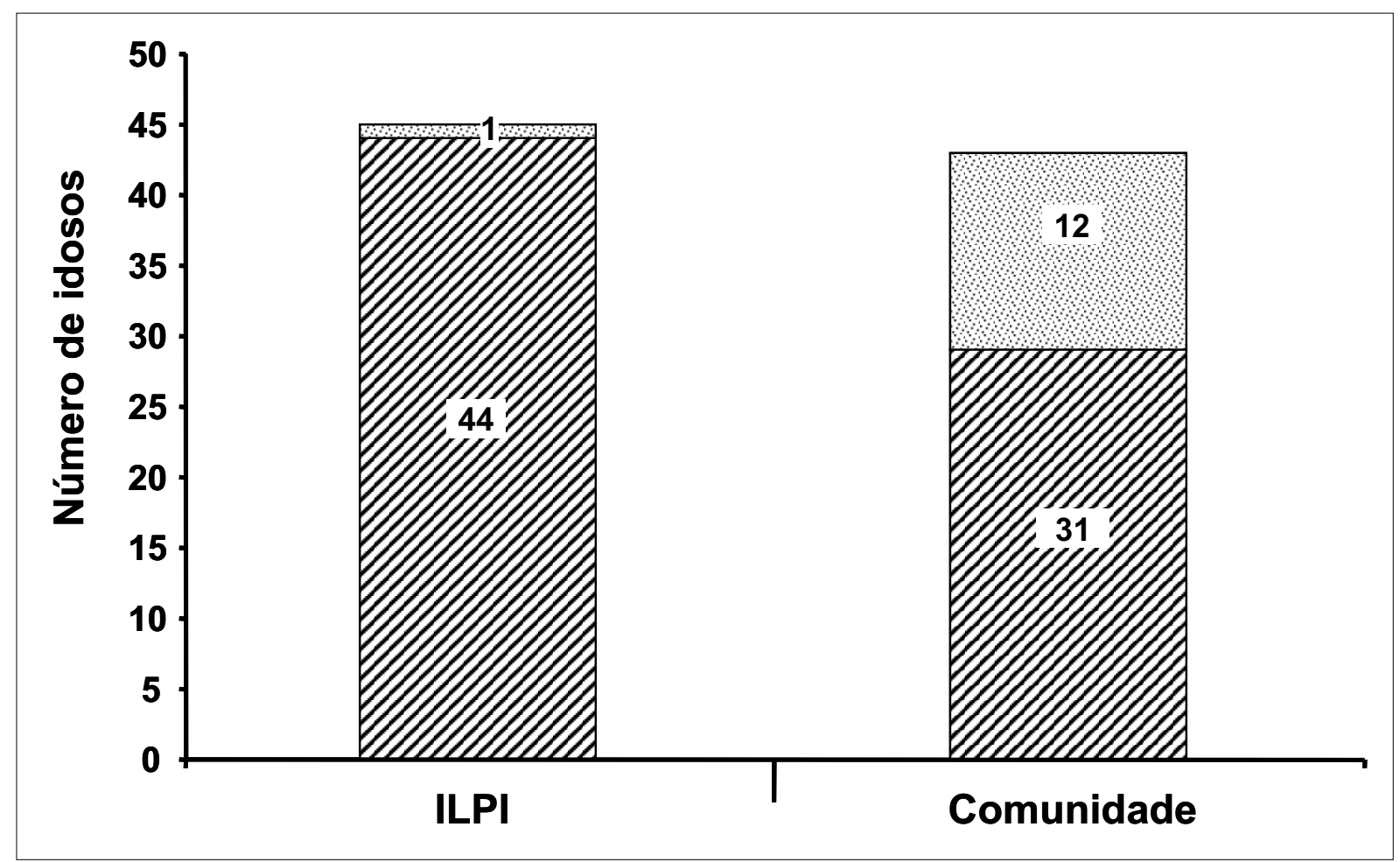

Figura 2 - Número de idosos com maior $(\mathbb{Z})$ e menor $(\square)$ risco de quedas em instituições de longa permanência para idosos - ILPIs - $(n=45)$ e na comunidade $(n=43) . R R=9,0 ; p=0,0001$.

\section{DISCUSSÃO}

Em vários países, as populações estão envelhecendo. O número de pessoas idosas cresce em ritmo maior do que o número de pessoas que nascem, acarretando um conjunto de situações que modificam a estrutura de gastos dos países em uma série de áreas importantes. No Brasil, o ritmo de crescimento da população idosa tem sido sistemático e consistente. ${ }^{14}$

O processo de envelhecimento está relacionado com a diminuição do equilíbrio, quedas, medo de cair, dependência, institucionalização e morte. No estudo, foi possível observar que o equilíbrio postural foi significativamente maior nos idosos da comunidade do que nos idosos institucionalizados. O controle do equilíbrio requer a manutenção do centro de gravidade sobre a base de sustentação durante situações estáticas e dinâmicas. Esse processo ocorre de forma eficaz pela ação, principalmente dos sistemas visual, vestibular e somatossensorial. Com o envelhecimento, esses sistemas são afetados e várias etapas do controle postural podem ser suprimidas, diminuindo a capacidade compensatória do sistema, levando a um aumento da instabilidade. ${ }^{15}$

Observa-se que quase a totalidade dos indivíduos moradores de instituições obteve escores 
abaixo de 45 pontos na escala de Berg, indicando risco aumentado de quedas. ${ }^{10}$ Através do cálculo do Risco Relativo, foi verificado que a população institucionalizada possui nove vezes mais chances de cair do que os indivíduos moradores da comunidade, segundo avaliação da Escala de Berg. Tem sido sugerido que idosos apresentam habilidade reduzida no controle postural, aumentando sua predisposição para a queda. ${ }^{16}$

Da mesma forma que no trabalho em questão, no estudo de Santos et al. ${ }^{3}$ foi constatado que a institucionalização representa um fator de risco para episódios de queda, já que esses idosos vivem em isolamento social e são mais inativos do que os idosos moradores da comunidade. $\mathrm{O}$ mesmo trabalho evidenciou que a grande maioria dos idosos residentes na instituição avaliada não realizava nenhum tipo de atividade física, o que, consequentemente, acelerou o processo de envelhecimento e o aparecimento de suas complicações, com perda progressiva da funcionalidade.

O estudo de Perracini \& Ramos ${ }^{17}$ concluiu que o comprometimento na realização das atividades diárias ou a inatividade são fatores de risco com alto peso de evidência para quedas em idosos. Tal fato pode ser observado também no presente estudo, sobretudo na população institucionalizada.

Uma maneira de aumentar o equilíbrio postural e diminuir o risco de quedas é a prática de atividades físicas. Exercícios com ênfase em

\section{REFERÊNCIAS}

1. Fabrício SCC, Rodrigues RAP, Costa Junior ML. Causas e conseqüências das quedas de idosos atendidos em um hospital público. Rev Saúde Publica 2004; 38(1):93-9.

2. Guimarães LHCT, Galdino DCA, Martins FLM, Vitorino DFM, Pereira KL, Carvalho EM. Comparação da propensão de quedas entre idosos que praticam atividade física e idosos sedentários. Rev Neurocienc. 2004; 12(2): 68-72.

3. Santos MLC, Andrade MC. Incidência de quedas relacionadas aos fatores de riscos em idosos institucionalizados. Rev Baiana de Saúde Pública 2005; 29(1): 57-68. mudanças de velocidade, amplitudes variadas, mudanças de direção e ambientes diferenciados, devem ser praticados. ${ }^{18,19}$

Em relação aos idosos institucionalizados, há um agravamento da situação, pois os mesmos dispõem de poucas atividades físicas podendo agravar o ciclo vicioso de envelhecimento, menor capacidade funcional, sedentarismo, atrofia e quedas.

Pelo fato de a queda poder ser considerada um evento sentinela na vida de uma pessoa idosa e como um marcador potencial do início de um importante declínio da função ou um sintoma de uma patologia nova, ${ }^{6}$ torna-se extremamente importante sua avaliação tanto em idosos institucionalizados, quanto nos nãoinstitucionalizados, para que políticas públicas sejam desenvolvidas na intenção de diminuir o risco de quedas. Tais políticas devem incluir a contratação de profissionais que trabalhem com a área, bem como o esclarecimento da população sobre o assunto.

\section{CONCLUSÃO}

Por meio da análise dos escores alcançados na Escala de Equilíbrio de Berg aplicada na população estudada, foi possível concluir que o equilíbrio de idosos institucionalizados é significativamente menor do que o de idosos não-institucionalizados. Foi possível concluir, ainda, que o risco de quedas é bem maior na população moradora em ILPIs.
4. Pinho L, Dias RC, Souza TR, Freire MTF, Tavares CF, Dias JMD. Avaliação isocinética da função muscular do quadril e do tornozelo em idosos que sofrem quedas. Rev Bras Fisioter. 2005; 9(1): 93-99.

5. Perracini MR. Prevenção e manejo de quedas no idoso. Portal Equilíbrio e Quedas em idosos. [Acesso em 15 jan 2008].Disponível em: http:// www.pequi.incubadora.fapesp.br.

6. Pereira SRM, Buksman S, Perracini MR, Py L, Barreto KML, Leite VMM. Quedas em idosos, projeto diretrizes. 2001 [ Acesso em 20 nov 
2008]. Disponível em: http://

www.sosvida.com.br/upload/Art_05.pdf.

7. Safons MP, Pereira MM, Rodrigues JFA. Efeitos do "Programa Melhor Idade Brasil Telecom" de condicionamento físico sobre a força dos membros inferiores de praticantes idosos. Rev Digital 2006 jul ; 11(98).

8. Gonçalves LG, Vieira ST, Siqueira FV,Hallal PC. Prevalência de quedas em idosos asilados do município de Rio Grande. Rev Saúde Pública 2008; 42(5):938-45

9. Faria JC, Machala CC, Dias RC, Dias JMD. Importância do treinamento de força na reabilitação da função muscular, equilíbrio e mobilidade de idosos. Acta Fisiatr. 2003; 10(3): 133-7.

10. Miyamoto ST, Lombardi Junior I, Berg KO Ramos LR, Natour. J. Brazilian version of the Berg balance scale. Braz J Med Biol Res 2004; 37(9):1411-21.

11. Vanswearingen JM, Brach JS. Making Geriatric Assessment Work: selecting Useful Measures. Phys Ther 2001; 81(6): 1233-52.

12. Ribeiro ASB, Pereira JS. Melhora do equilíbrio e redução da possibilidade de queda em idosas após os exercícios de Cawthorne e Cooksey. Rev Bras Otorrinolaringol. 2005, 71(1): 38-46.

13. Figueiredo KMOB, Lima KC, Guerra RC. Instrumentos de avaliação do equilíbrio corporal em idosos. Rev Bras Cineantrop Desempenho Hum 2007; 9(4): 408-13.

14. Instituto Brasileiro de Geografia e Estatística (IBGE). Síntese de Indicadores Sociais. Uma análise das condições de vida da População Brasileira. 2010. [Acesso em 21 fev 2011]. Disponível em: http://www.ibge.gov.br/home/ estatistica/populacao/condicaodevida/ indicadoresminimos/sinteseindicsociais2010/ SIS_2010.pdf.

15. Ruwer SL, Rossi AG, Simon LF. Equilíbrio no idoso. Rev Bras Otorrinolaringol. 2005;71(3):298-03.

16. Melzer I, Benjuya N, Kaplanski J. Postural stability in the elderly: a comparison between fallers and non-fallers. Age Ageing. 2004;33(6):602-7.

17. Perracini MR, Ramos LR. Fatores associados a queda em uma coorte de idosos residentes na comunidade. Rev Saúde Pública 2002: 36(6): 709-16.

18. Goulart F, Chaves CM, Vallone MLDC, Carvalho JA, Saiki KR. O movimento de passar de sentado para de pé em idosos: implicações para o treinamento funcional. Acta Fisiatr. 2003;10(3):138-43.

19. Alfieri FM, Teodori RM, Montebelo MIL. Mobilidade funcional de idosos submetidos a intervenção fisioterapêutica. Saude Rev 2004;6(14):45- 50. Disponível em: http:// www.unimep.br/phpg/editora/revistaspdf/ saude14art06.pdf. 